\title{
The Future of IR: Ascending! Keynote Lecture at the Pairs Annual Scientific Meeting 2017, Dubai, UAE
}

I cannot tell you what a thrill it was for me to be in here and an honor (at the Pairs Annual Scientific Meeting 2017). As someone who has been a passionate about education and spreading the words so to speak regarding Interventional Radiology and less invasive therapy, in general, it is really exciting to see what it is going on in this region, having sat through lectures yesterday and today again. Everyone here should be really proud of what is happening right here in this region. For me, as an educator and someone who has tried to take the message of those early years and spread it worldwide, it is a lot of personal satisfaction, so congratulations.

I was given this title in talking about the future, I will shorten it a little bit regarding "ascending" and in many ways, Interventional Radiology, to many people, it's already arrived, it's already gotten there already. However, actually to me, it has always been about the future and about where our platforms can take us and where we are going. People who work close to me know I live in a window that is about 5 years down the line. It is frustrating to people who are close to me because sometimes I do not pay attention enough to the present, and so I am going to try to give a little insight in some of the big pictures, and there were so many great lectures that have occurred already in dealing with new technologies and new approaches to critical limb ischemia (CLI), peripheral artery disease, and aortoiliac disease. I am not going to dwell so much on that but try and give you a big picture.

At this year's SIR meeting, there was an interesting talk given by my Mike Darcy, who is the Dotter Lecturer this year. As he is talking to his fellows, he gave his fellows a quiz with some of the big names so to speak of Interventional Radiology, mixed in with names of famous performers, artists, musicians, or whatever that was, so there was Cesare Gianturco and there was a Cesar Romero, and they had to pick which one is an interventional radiologist and are they dead or alive and what did they do. He showed this as part of the Dotter Lecture. I was pretty pleased to see there were three interventional radiologists that all of them recognized. I was only recognized by $90 \%$ of the fellows, but the good news is they thought everyone else is dead, including Ernest Ring, but at least 90\% thought I was alive, so it was good.

In my perspective, I want to change my presentation today a little to talk a little bit about the past so that you would understand not only some of greats and how we got to where we are but also how important step-by-step technology is and every incremental step we make is an opportunity for the future. It is only one step moving on with to the future.
Hence, in talking about this subject, I think it is important to understand what IR is and I spent a good part of my career trying to change my identification because, in my institution, I actually lead the cardiac surgery program and the interventional cardiology program. We integrated all services up to a final encounter pathway, but I found it actually to be unsuccessful and today I think we are interventional radiologists and so it is best if we train that way and we, of course, do image-guided procedures that are therapeutic in nature, but importantly, we provide clinical care to patients. This is probably the most important thing we do, and we do it across diverse group of procedures, which differentiates us from many in Medicine.

We are a discipline that is spread throughout the world, as you see here in this meeting, offering patients less invasive solutions to significant medical problems, and we are all about innovation and change to health care, and that is one of the things that are different between us and traditional vascular surgeon, traditional endoscopies, or traditional anyone that is involved in less invasive therapy. Most interventional radiologists at their core are looking forward to change in innovation.

Hence, I think our specialty is generally considered and has been worn when Charles Dotter, not just did the first angioplasty that was obviously a very significant event, but more importantly when he published this first paper and he talked about two important things; one is using the catheter as a surgical instrument and so most of us in Intervention Radiology are, in fact, surgeons. Many of my fellows call themselves minimally invasive surgeons or endovascular surgeons in the United States, but importantly from this little experience, he thought enough to forget all of the things that you see here in this slide and virtually all of which has become true.

Hence, if we look at the impact that Interventional Radiology has had on Medicine, our general space. Virtually everything on this list has been totally changed by Interventional Radiology. Some surgery has been totally eliminated. When I was a radiology resident, the most common surgical procedure in the United States was exploratory laparotomy. It is pretty amazing when you think about it and today, you know, it is unheard of, we can figure everything out with image-guided biopsies and sophisticated imaging, feeding problems, peripheral pulmonary AVMS, uterine fibroids, colonic bleeding, subarachnoid hemorrhage, and coronary artery disease.

All these are the things that we have had a very dramatic impact on and, in fact, Vascular and Interventional 
Radiology propelled this entire movement in Medicine toward less invasive therapies whether it be laparoscopic, endoscopic, or neurovascular, and many of the people that came from our early circles in the industry are the ones that founded these various companies to go out and create laparoscopic approaches, endo any kind of approaches.

Hence, we have developed as a specialty over the past 40 years, and in the United States 3 years ago, achieved subspecialty status, actually specialty status, with accreditation by the American Board of Medical Specialist. This has been a scary thing, I note to many of my colleagues in Europe, who maybe do not agree with this direction but that is the direction that we, in the United States, feel really important to define what IR is. Vascular therapy has been a cornerstone of intervention, but in many ways, it was just a platform for us to spread out and use these techniques and other types of applications. When I started, virtually all cardiac catheterization was done by radiologist and so, one of the reasons I am particularly in a place where I am leading the cardiovascular program as I was old enough to basically have cardiovascular experience that most radiologists do not today, but that transitioned of course, and once balloon angioplasty developed, our cardiology colleagues became much more interested in this space.

Hence, if we look back in the earlier years of intervention when the term Interventional Radiology began to develop, you can look at kind of the procedures that were the "bread and butter" of procedures, and this is what everybody's workday looks like. However, things have really dramatically changed and one of the things I think all of us understand is that, the procedures that we do today, most that are important, most of them did not exist 10 years ago or 12 years ago, you pick the time and so, the nature of Interventional Radiology, from an educational point of view, is we have to train our fellows to do procedures that do not exist. This is to give them the skills, to give ourselves the skills, and the mental strength to be able to do things that do not exist because that is our nature.

These are some early papers. You can see in 1979, we were publishing early balloon angioplasty, I thought I would share some of these with you for historic purposes in hypertension in 1979.

Right from the beginning, myself and you will see Dr. Dake's name, there who was a partner of mine, early in his career, began to understand that we had to take this evolving specialty and become significant, not just be a practitioner; and to be significant, we had to become a clinical discipline. It was pretty obvious that we were the only people in Medicine who were not taking care of our patients. Everybody else is taking care of the patients that they did procedures on, and coming from a clinical background myself, it just really did not make any sense.
Hence, we began to write along with others about the importance of clinical training.

Finally in the United States, we see this actually being formally structured as the US training pathways are changing. As you know, there is going to be a residency in Interventional Radiology now, meaning the fellowship are going to go away, and these residencies are going to include time in critical care, time on the floors, and clinical management, and refreshing the skills or adding to the skills that we need.

Today, in modern IR, hospitals at least in the United States cannot function without an interventional radiologist or more, and there are hospitals that have lost their IRs over turf battles and things like that, and they have been written up in journals and created emergencies for patient care. It is really pretty amazing how we are woven into the metric of day-to-day practice.

Again, Interventional Radiology has accomplished so much. If you were to look at the curriculum vitae of our specialty and what we have accomplished whether it is in peripheral arterial disease, cancer treatment, palliative care, and treatment of aneurysms. However, things have changed over the past 20 years, and what we have found is that we have developed and we practised procedures that other people want to do and the people who want to do them are the disease management doctors. They are, you know, somebody who was trained on how to take care of vascular disease and want to be able to do the procedures associated with taking care of his or her patients and so this has been a challenge for our discipline. We have been able to figure it out in Miami and survived in a significant way.

It has been a challenge to IR as we have been unable, in many cases, to hold on to our innervations.

Hence, I just want to talk a little bit about some megatrends and challenges. Now, instead of being a developing field, endovascular therapy is really a mature field. It is very broad. It is very important for us to define who benefits and bring science to this discipline. Some of them were cardiology and vascular surgery.

One of the other megatrends has been the effect of computed tomography angiography and magnetic resonance angiography, so our trainees no longer have diagnostic angiography to use as a platform for developing basic catheter skills. They are expected just as surgeons to go from zero into doing some sort of complex recanalization; it is pretty challenging from a training and educational point of view. We obviously are defining now the role and methods of drug delivery, and we are experiencing a worldwide in epidemic CLI.

Hence, it is uncommon in us, especially in these regions and other regions of the world, to make endovascular therapy more cost-effective and less expensive both, and how are we going to do this. I am going to talk more about amputation 
rates and some of the public health issues, but more and more, Interventional Radiology is challenged now to produce proof of our procedures. When we did the first angioplasty, it was pretty intuitive, "Wow! This patient can walk now and did not need surgery." Well, that no longer gets us anywhere and the compelling need for data in clinical trials is important and you will hear that I talk about our failures and some of the new procedures that you will see here are going to need data and proof before our colleagues accept it.

We look at endovascular as very broad, so to me treatment of an AVM is endovascular, pretty much anything if we take the number 2 broad definition and how we, ourselves, are organized, and it is any discipline, any organ system, anything that involves image-guided therapy. However, others can look at it in a different way, they can look at it, to many vascular surgeons, endovascular means aortic work and somehow peripheral intervention means something different. Hence, we still have a lot of definitional issues within the entire space.

If we look at IR today, specifically endovascular therapy has replaced much traditional vascular surgery, but it has also become cardiovascular surgery. In the United States, about 30 percent of this is done by interventional radiologist and has become a multidisciplinary field. That is just the way it is, it is steady but it is multidisciplinary, and so interventional radiologists, among other things, needs to learn how to compete.

With endovascular therapy, we can do a lot of things, opening vessels, closing vessels, and treating cancer; it is all part of using the circulation as a therapeutic conduit. The pioneers in this space really started in the 60's; these are Stanley Baum and Josef Rosch, who unfortunately both passed away and the concept of using the catheter for surgical tool, in this case to deliver drugs, was developed by them. The concept of trying to get a balloon to make an artery bigger than the catheter itself is generally credited to Werner Porstmann who, at that time, was behind the iron curtain in Charite Hospital in East Berlin, and I thought I would share this with you, this is actually the first patient I treated in Rome; it was in 1974, and I am the one with the beard and the big hair along with the others, but this patient did incredibly well with that corset catheter for 6 or 7 years we had angios on this patient, it was amazing what could be done and this is what fired our whole discipline, followed by Gruentzig who developed the technology to reach his dream of treating coronary arteries, and along the way, incorporated the vascular system as well, and then, a group of people took the next step which was opposed by Dotter actually of managing thrombus and managing the clot with a catheter and then stents came along, and the thing about these and the reason that I am going through this is that each of these was one step going to the next.

Hence, stents were interesting as you can see, but maybe there were more important to develop a platform for endografts and the concepts of endografts or the concept of drug delivery. Much more important, drug delivery or the stent and so each of these incremental concepts were very important in moving our field forward.

Now, we still have a ways ago, people are dying of aneurysms. These are some of the well-known people that have passed away from ruptured aneurysms. Some of them knew they had aneurysm, some of them did not and we know that aneurysms can occur everywhere, and of them we knew had them some of them we did not, we know that we have made a great stride here but also we have taken something that was an idea and made it standard of care.

And so, each of these areas that we have touched on, we have actually made standard of care and each and every one of you are part of a field that should take great pride in that. It has not all been perfect and one of the things you have to know about innovation is that innovation involves failure, and these are some of the devices that have failed. Some types they fail early, some types they fail late, but we have to be careful when we talk to our patients, in particular, to be intellectually honest about what we know and what we do not know and some of the devices, I am not sure if some others which have failed. However, where are we trying to take endovascular aneurysm repair (EVAR)? The fact that we can treat an aneurysm less invasively, that is not the end-game; that is the beginning. The end-game is for patients to be able to come in, have their aneurysm fixed and go home the same day. That is dramatically changing in the way healthcare is delivering. We have been very interested in this for a long time, and it is not just going to involve the technology or the right piece of equipment, it is going to involve a total change in how we manage patients and how we get paid for patients. In the United States for instance, if you were to treat a patient as an outpatient, you cannot get paid; it is required to be an inpatient. as a result, this particular study, the LIFE study was done on inpatients that simulated outpatients, and they all had percutaneous access, no general anesthesia, no ICU and were pushed for next-day discharge with 250 patients and this was recently presented at ISET and has been presented in other meetings as well, and you can see that comparing it to that analysis as traditional EVAR, that there was great safety, reduced procedure time. The length-of-stay was 1.2 days because we were not allowed to send them home basically during the course of this trial, and importantly, the readmission rate was much, much lower at 30 days than traditional EVAR patients, so there was no intent to push these patients out only to have them be readmitted in a short period.

Here, you can see the performance goal in terms of major adverse event (MAE) rates, and you can see that of the 250 patients in a so-called fast track, there was only one that had any type of MAE and that was a readmission in 30 days.

Along the way, you are going to hear more about during this meeting. As image-guided therapist, imaging has been 
of significant improvement to us as we can combine what used to be just looking at a flat screen like this and learning how to do this in our heads to be being able to superimpose $3 \mathrm{D}$ models, and this is not just for the uneducated or people that are not radiologists. This technology is making all of us better by able to see precisely and understand what we are doing and nowadays with image fusion, we can actually move the table and around rotate and keep everything synced up and so for doing very complex cases, it has really been a significant improvement.

One of the important areas of Interventional Radiology that is becoming more important is the issue of CLI, and there were a lot of talks yesterday, great talks about how to treat CLI but I am not going to talk about that. What I want to talk about is CLI as a disease and point out that $25 \%$ of our patients with CLI are going to be dead in 12 months, probably $70 \%$ are going to be dead in 3 years and so similar to what we did when we started treating SFA disease, it is really important that if you are thinking about developing a CLI program, that you start thinking about the patient as a whole, not just of the wound and yesterday, we saw some cases where revascularization was made but the patient lost their leg because of lack of teamwork and lack of combined therapy that is necessary. Hence, we know that is an epidemic and pandemic and we think that there is a great opportunity from the public health point of view.

Now, all ideas are not just technology. We have some great thinkers in our space, one of them being Dr. Manzi, who is on the program this year who thought about approaching things on these very tiny arteries in the foot that most of us thought were impossible. In fact, I heard someone yesterday during the comment section, and the idea of combining retrograde and antegrade access to accomplish something which could have been a failure before or to avoid failure is an example of how creative interventional radiologists are in creating unique solutions.

In this particular case, I think by now probably all of you had experienced here, you can act up immediately and it is very helpful in this particular case, myself and my vascular surgical colleagues are working together, and teamwork is very important to get greater results here.

Hence, all ideas and not technology or many of them, especially in CLI, come from our brains without a lot of expensive thought or technology. Hence, what is CLI? Well, we did a survey in the United States and if you survey 10 people what CLI is, you get 10 different answers, and what that means in the United States at least is that we cannot get paid for it. Hence, if a patient comes in with CLI and we are trying to figure out how much does it cost to take care of a patient with CLI, it is very, very difficult because there are no codes, there is no identification, and we are very interested in developing a CLI program to prolong life and reduce amputations.
So for instance, if you were to look a Rutheford 6, these are three different feet. They certainly do not have the same probability of success in terms of saving that foot and yet, right now, we do not have any way of really classifying those differences, and so there is really a critical need to do that.

Recently, in the United States, the Society of Vascular Surgery has proposed the WIFI system, which would allow us to be able to differentiate what the classifications are. What we know is that $50 \%$ of the patients that actually get revascularization, $25 \%$ are going to be dead in a year, $30 \%$ are still going to have amputations of some sort, and only $25 \%$ are going to have their CLI resolved. Hence, interventional radiologists want to look for opportunity. We generally want to create procedures and technologies that fill gaps but to do that for CLI, we need to develop metrics for success, and we need a definition of patient population.

If you compare us to ST-segment elevation myocardial infarction, for instance, in acute myocardial infarction, we are so far off of the metrics necessary to really make an impact on CLI and that there is a lot of work to do. And so, this year at ISET, myself and a number of others announced the formation of the CLI Global Society. Those of you who are involved in this hopefully will get involved in this phase, but this is not a society that has meetings and things like that. The mission is to improve the quality of life by preventing amputations and death due to CLI, so we actually want to take that mortality rate and lower it. We want to take amputation rates country by country and lower it, similar to what the American Heart Association has been doing for cardiac mortality.

The next technology I want to briefly talk about are things that are going to be important. We know about drug-eluting balloons and drug-eluting stents, but Bioabsorbable Scaffolds are the next hot thing, leaving nothing behind and a lot of people believe this is the next great thing. However, in the past year, it has become clear that there are significant issues related with this technology that is probably going to bring some caution. One is that generally, they require OCT to be able to determine whether you got good wall apposition. Most interventional radiologists do not have OCT as an integral part of the laboratory. That also means slow cessation to be able to image it. However, the imaging is exquisite of course. I think it is certainly true that device-drug combinations are ultimately going to be part of what we treat all patients with, in my opinion, but I think regarding Bioabsorbable, all we can say is that they may play a role today but they are not the answer yet.

Now, I mentioned also that in pushing for change and pushing for innovation, we have made some mistakes and we have some procedures that have failed and they have had an impact on failure and this year at SIR, two important trials were released. One is the ATTRACT trial which is a randomized trial of iliofemoral thrombolysis. 
There are a lot of problems with this trial by the way, but the end-point was venous stasis syndrome and it turned out to be no difference at 2 years. Morever, the other one was on multiple sclerosis.

How many people remember what the frenzy was like 5 years ago with centers opening up, many of them in this region to treat these patients, and now what is happening is too many interventional radiologists were really unfortunate carrying the flag on this. Most of us were skeptical but others were not, and a lot of patients got procedures they did not need as a result.

Renal denervation is another example. Multibillion dollars spent on technology and then a single trial which was misinterpreted, in my opinion, more or less killed an entire space of research which continues on.

Hence, how about isolated organ perfusion? Anybody involved with that? I think there were some in this region that were great promise, probably the wrong drug, the wrong cancer, and it wound up being a failure. So as IRs matured, we know that there have been some failures that we have had and we have to be prepared for that.

Now, what final thing I want to mention is what about threats to us. Well, we may not have heard about Tom Lawson, he is a scientist. He used to be at Duke University, but I think he left to join this company. However, he basically figured out how to manufacture artificial blood vessels, and they are actually being put in patients in Eastern Europe now.

Now, if you can make a blood vessel and you can deliver drug out of that blood vessel, you know, then think about that as it relates to stents and the things we do. This could be a potential game-changer for all of us.

Robotics, I think you all know what happened to robotics. Unfortunately, myself and Professor Hamady, we believe that there is a future there but I am not going to dwell on it because of recent events, but we do believe that there is an opportunity.

And then finally, I think, on a positive note, what are the next great areas that we are going to be involved in and one of the big emphases is now on pulmonary embolism. It is a very common cause of death in hospitalized patients, and there is big initiative that is welled up now to look at submassive and massive pulmonary embolism and the role of interventional therapy. These are very dramatic things. In this particular patient, a catheter was put in the main pulmonary artery, just maceration by spinning the catheter followed by catheter-directed lysis in this case with ultrasound enhancement and you can see the images on the lower right. But most importantly, if you look at the right ventricle-to-left ventricle ratio before and after, you can see that this has had very significant effect on cardiac function. The reason that patients with PE die is because of acute right heart failure generally. They die reflexively and deal of acute right heart failure. Hence, these are potential life-threatening initiatives, and the development of pulmonary embolism response teams is a big initiative.

There are some other out-of-box applications I think you are aware of, prostatic embolization. People are very, very excited about that. We can see the prostate shrink. How could this possibly not work? How could CCSVI not work? How could any of these other things not work? We need clinical data and we need trials and those of us in the space should be demanding it, same thing with left gastric embolization for morbid obesity. Very exciting!

Then, finally, Interventional Oncology this is the great savior of Interventional Radiology. I gave a talk at a guest meeting 2 years ago on the death of Interventional Oncology, so the same thing can happen if we do not embrace clinical management of patients. Hence, if you look at this, this is clinical Interventional Radiology, technology, and multidisciplinary care has to interact. Hence those of you, who are engaged in this space, need to be able to be prepared to learn a lot about cancer, a lot about longitudinal management. This is not just about picking the right way to kill a cancer focally but managing the morbidity long term as a result of that. When you start giving radiotherapy in any type of way, you need to be prepared to manage those patients longitudinally and prospectively in terms of providing input on patient safety and long-term morbidity.

We know that our space is seeing a lot of imaging, you can hear more a about that, and the final thing that I want to share with you is this idea:

What if you can put in stent in someone and when that stent starts narrowing, you get a warning on your iPhone or in your office that that stent is starting to fail. Well, this is the technology we are beginning to look at in the case of both bypass grafts in this model for dialysis fistulas. This was also presented at ISET that first-in-man trials are getting ready to be run. But you can see, here is the input on animals who had dialysis grafts put it and then a stenosis was created, you can see the follow-up on the image on your lower image.

While for most of us interested in bypass graft is less, they can manufacture and put this piezoelectric sensor actually into a multisegmented stent which, in theory, could provide us feedback and reduce the need for a lot of other studies and then send the signal back to an iPhone.

So what about the future? Well, for those of you living and working in this region, I tried to get some specific information, and this is a transient forecast on the business side of things and will forecast out through 2024.

In the inner core, there is the Middle East and Africa, the smaller one, which I think is actually an underestimation 
but you can see the relative growth in different regions of the country but I think this is a global mix of different types of cases and given our impact on medicine, I see no way that it is not going to grow significant in this region. If you look at this business analysis in terms of where that growth is going to occur, a lot of us projected in still in arterial lower extremity revascularization, it is the big bubble just above coronary there, in this region as well, as well as venous intervention and then, there are some other AAA recurrence so on by the smaller bubbles.

Hence, think about it, how many procedures do you do today that you did 10-15 years ago? Probably, a lot but you did not do, most of them are new. What are you going to be doing 10 years from now? Likely, new procedures that are being developed and I would mention a little bit, but are being proven today and one of the things you need to worry about is creating the environment to do these procedures better and especially here in an environment where things are very cost sensitive and we rely on expensive technology. Hence, we need to work together with our industry partners to figure out all the solutions to provide such valuable care to patients that you take care of or patients that you need without unlimited capital. You know, it is definitely a challenge because we have a better way to take care of patients than the status quo.

Hence, I say, continued growth worldwide driven by technology and ideas that are going to continue innovation, Interventional Radiology is going to begin to prove cost efficacy. In the United States even, we are on a huge pressure to every clinical trial we do, has to have a health-care economics model to it and the FDA is starting to require cost-benefit not just a clinical benefit. As technology gets more broadly adaptive, cost will decrease but also competition is going to increase and training of the next generation interventional radiologist will continue to be difficult because of the change in procedural mix that we are constantly facing.
Hence, in conclusion, we are very excited to be part of a growing specialty of medicine which impacts virtually every disease process wherever you live. There is some disease that is going on that we can help. It is interesting that it is junction between technology and disease as where we sit led by physicians who are interested in changing that status quo, not simply diagnosing and treating disease. As we grow as a specialty, filling more clinical services will be expected by others, they are going to expect us to care of these patients and remain necessary to compete as well with disease management specialists who are interested in doing the same procedures.

Hence, with that, I thank you very much for your attention. I hope some of you might be able to come and visit us next year in Miami or South Florida and thank you very, very much for your attention.

Barry T Katzen

FIU Herbert Wertheim College of Medicine, Miami Cardiac and Vascular Institute, Baptist Hospital of Miami, Miami, FL 33176, USA.

E-mail:Btkatzen@aol.com

This is an open access article distributed under the terms of the Creative Commons Attribution-NonCommercial-ShareAlike 3.0 License, which allows others to remix, tweak and build upon the work non-commercially, as long as the author is credited and the new creations are licensed under the identical terms.

\begin{tabular}{|l|l|}
\multicolumn{2}{|c|}{ Access this article online } \\
\hline Quick Response Code: & Website: \\
& www.arabjir.com \\
\cline { 2 - 2 } & DOI: \\
\hline
\end{tabular}

How to cite this article: Katzen BT. The future of IR: Ascending! Keynote lecture at the Pairs Annual Scientific Meeting 2017, Dubai, UAE. Arab J Intervent Radiol 2017;1:43-8. 